\title{
Trabajo cooperativo en grupos de tutorías de Aditivos Alimentarios
}

\section{Francesc A. Esteve-Turrillas}

Departamento de Química Analítica, Universitat de València, Edificio Jeroni Muñoz, c/ Dr. Moliner 50, 46100 Burjassot, España (francesc.a.esteve@uv.es)

\begin{abstract}
In the present communication an strategy based on the Puzzle of Aronson has been applied to promote the cooperation in working groups in the tutorials of Food Additives course of the Degree in Science and Food Technology in the University of Valencia. This tool allows the training of transversal skills in the classroom such as: social and communication skills, decision making, and conflict resolution. A rubric has been applied to evaluate the cooperative work process and the obtained results have been discussed. Finally, we include data on the student satisfaction with the proposed activity through the completion of a survey.
\end{abstract}

Keywords: Cooperative learning, Aronson Puzzle, Group work, Food additives, Evaluation

\begin{abstract}
Resumen
En la presente comunicación se presentan los resultados obtenidos en el empleo de técnicas basadas en el Puzle de Aronson para fomentar el trabajo cooperativo en la asignatura Aditivos Alimentarios del Grado de Ciencia y Tecnología de Alimentos de la Universitat de València. Con esta herramienta se consigue trabajar competencias transversales en el aula relacionadas con el desarrollo de habilidades sociales, comunicativas, toma de decisiones y resolución de conflictos. Se ha aplicado una rúbrica para evaluar el proceso de trabajo cooperativo y se han discutido los resultados obtenidos. Finalmente, se incluyen datos del nivel de satisfacción de los alumnos con la actividad propuesta a través de la realización de una encuesta.
\end{abstract}

Palabras clave: Aprendizaje cooperativo, Puzle de Aronson, Trabajo en grupo, Aditivos Alimentarios, Evaluación

\section{Introducción}

El aprendizaje cooperativo se centra en la teoría constructivista que otorga un papel fundamental al alumno, el cual es el actor principal de su propio proceso de aprendizaje (Kagan, 1994). Una situación cooperativa consiste en que un grupo trabaje para alcanzar objetivos comunes que sean beneficiosos tanto para ellos mismos como para los demás miembros del grupo. De la misma forma la responsabilidad para la consecución del objetivo propuesto es compartida por todos los miembros del grupo. Resulta importante 
destacar la diferencia con el aprendizaje competitivo en que cada alumno trabaja en contra de los demás para alcanzar objetivos escolares individuales, o con el aprendizaje individualista, en el que los alumnos trabajan por su cuenta para conseguir objetivos escolares desvinculados a los demás compañeros (Johnson, 1994). Existen cinco elementos clave que se deben cumplir para asegurar que la cooperación se realice adecuadamente (Johnson, 1991).

- El primero de ellos consiste en la interdependencia positiva, que consiste en que grupo debe de hacer frente a un problema o resolver una situación que requiere la participación de todos los miembros del grupo. Del mismo modo, el beneficio obtenido no será individual, sino que beneficiará a todos los integrantes del grupo. Esta interdependencia entre los miembros del grupo fomenta el compromiso con el éxito de otras personas.

- El segundo elemento a cumplir consiste en la responsabilidad tanto individual como grupal. El grupo asume la responsabilidad de alcanzar sus objetivos, y cada miembro del grupo será el responsable de cumplir su tarea concreta de una forma autónoma, adquiriendo las competencias necesarias para realizarla. Asimismo, el individuo es responsable de comunicar al grupo los resultados obtenidos de una forma eficiente.

- El tercer elemento consiste en la interacción estimuladora. El grupo realiza juntos una tarea, en la que cada miembro debe promover el éxito de sus compañeros, compartiendo recursos, ayudándose, animándose, y felicitándose unos a otros por su empeño en aprender. Al participar activamente en el aprendizaje de los demás, los miembros del grupo adquieren un compromiso personal entre ellos, constituyendo un sistema de apoyo y de respaldo personal.

- El cuarto elemento consiste en guiar a los alumnos en la aplicación de prácticas interpersonales y grupales necesarias para funcionar como parte de un grupo de trabajo cohesionado, como ejercer la dirección, toma de decisiones, crear un clima de confianza y motivación, y sobre todo manejar los conflictos. Ya que las técnicas cooperativas promueven la práctica de procedimientos y técnicas para manejar de una manera constructiva los conflictos que se generen.

- Finalmente, el quinto elemento esencial en el trabajo cooperativo consiste en la evaluación grupal, donde los miembros del grupo analizan la consecución de las metas propuestas. En esta parte el grupo debe decidir qué acciones de sus miembros son positivas o negativas, y tomar decisiones para continuar o modificar estas conductas. En este sentido, el hecho de analizar los resultados obtenidos por el grupo durante la actividad y la propuesta de cambios en el plan inicial permite aumentar su eficacia.

El papel del profesor durante la realización de la actividad es fundamental ya que durante este proceso el profesor debe supervisar el correcto desarrollo de la actividad para guiar a los alumnos en la consecución del trabajo propuesto de forma cooperativa siguiendo las cinco pautas mencionadas anteriormente.

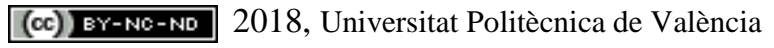

Congreso IN-RED (2018) 
La técnica del puzle de Aronson permite de una manera simple, dinámica y funcional promover el trabajo cooperativo en grupos de trabajo. En dicha estrategia de aprendizaje los estudiantes trabajan en grupos constituidos por un número de integrantes no superior a cuatro personas, en el que se realiza un reparto cooperativo de tareas (Martínez, 2010). Cada alumno se especializa en un tema y lo debe transmitir al resto de compañeros para ayudar a resolver un problema común propuesto. La propia estructura de la estrategia de puzle de Aronson promueve la generación de conflictos, cuya superación consolida, fortalece, prepara y anima al grupo para la resolución de futuros conflictos. Las habilidades que se trabajan y promueven en el desarrollo de actividades centradas en estrategias del tipo puzle de Aronson se basan en: 1) mejorar el aprendizaje cooperativo; 2) desarrollar habilidades sociales, asertivas y de comunicación; 3) desarrollar la solidaridad y el compromiso cívico entre el alumnado; 4) fomentar la autonomía en el aprendizaje; 5) aumentar el rendimiento académico; y 6) desarrollar competencias académicas y profesionales (Traver, 2004).

\section{Objetivos}

En la edición anterior de IN-RED 2017 se presentó la propuesta de implementación de una estrategia tipo Puzle de Aronson en las tutorías asistenciales de la asignatura Aditivos Alimentarios del Grado de Ciencia y Tecnología de los Alimentos de la Universitat de València (Esteve-Turrillas, 2017). A través de esta dinámica de trabajo de grupos se trabajan competencias relacionadas con el aprendizaje cooperativo, se desarrollan habilidades sociales y de comunicación, y se fomenta la autonomía en el aprendizaje. En esta segunda comunicación se aportan los resultados obtenidos en el curso 2017-2018. En primer lugar se evaluó la adecuación de la actividad propuesta para desarrollar el trabajo cooperativo de grupos a través del empleo de una rúbrica. Los resultados obtenidos a partir de dicha rúbrica se discuten en la presente comunicación, así como la valoración del estudiante sobre la dinámica realizada a través de una encuesta de opinión. Finalmente, se han comparado las calificaciones obtenidas en el presente curso, con aquellas obtenidas en el curso 2016-2017.

\section{Desarrollo de la innovación}

\subsection{Contexto de aprendizaje}

La técnica de trabajo cooperativo se ha aplicado por segundo año consecutivo en la asignatura Aditivos Alimentarios correspondiente al Grado de Ciencia y Tecnología de los Alimentos que se imparte en la Facultad de Farmacia de la Universitat de València. La asignatura incluye 25 horas de teoría, 15 horas de prácticas de laboratorio, 2 horas de seminarios coordinado y 2 horas de tutorías presenciales. La asignatura es optativa del grado y se imparte en cuarto curso, con una frecuencia de 2 horas semanales. El número de alumnos en el curso 2016-2017 fue de 33. La asignatura se imparte en un aula con asientos y mesas fijos con una capacidad de 120 personas. El perfil de los estudiante en el curso 
2017-2018 es el siguiente: 69.7 \% mujeres y 30.3 \% hombres, edad promedio de 24.4 años, un $93.9 \%$ no realiza ningún tipo trabajo remunerado. El $57.6 \%$ proceden de P.A.U., el $24.2 \%$ de un ciclo formativo superior, el $12.1 \%$ de programas internacionales de intercambio (Erasmus), el 3.0 \% de pruebas de acceso para mayores de 25 años, y el $3.0 \%$ traslado de otro grado.

\subsection{Contenidos de la asignatura}

Como indica la guía docente, los contenidos que se imparten en la asignatura Aditivos Alimentarios se dividen en 13 temas, correspondientes a las distintas categorías de aditivos alimentarios que se emplean y están permitidos en la Unión Europea. Asimismo se incluye un primer tema de introducción, uno de evaluación toxicológica y finalmente uno de legislación actual relacionada con el área de conocimiento. A continuación se indica el título de los temas impartidos, así como el número de horas asignado a cada tema.

Tema 1. Introducción (1 h)

Tema 2. Aditivos antioxidantes (3 h)

Tema 3. Aditivos antimicrobianos (3h)

Tema 4. Agentes depresores de la actividad de agua (1h)

Tema 5. Antiendurecedores (2 h)

Tema 6. Aromatizantes y potenciadores del sabor (2 h)

Tema 7. Edulcorantes (2 h)

Tema 8. Colorantes (2 h)

Tema 9. Espesantes y gelificantes (2 h)

Tema 10. Emulgentes (1 h)

Tema 11. Auxiliares tecnológicos de fabricación (2 h)

Tema 12. Evaluación toxicológica (2 h)

Tema 13. Legislación relativa a aditivos (2 h)

\subsection{Temporalización de la actividad}

La presente actividad se ha realizado en dos sesiones de tutorías presenciales en el aula (1 hora cada sesión) que se realizó a mitad del primer semestre. Ambas tutorías se realizaron en un plazo de dos semanas. Durante el tiempo transcurrido entre tutorías, el alumno complementó la información, buscando contenidos adicionales fuera del aula (biblioteca, Internet, visitas a supermercados) para preparar la actividad a realizar en la segunda tutoría.

\subsection{Descripción de la tarea}

En las tutorías de esta asignatura se desarrolló una actividad basada en el puzle de Aronson para fomentar el trabajo cooperativo de grupos. Los alumnos se dividieron en dos grupos de 16 personas aproximadamente para realizar las tutorías, ya que el número de alumnos fue relativamente reducido. La actividad propuesta consistió en la resolución por

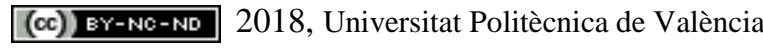

Congreso IN-RED (2018) 
grupos de trabajo de diferentes problemas prácticos relacionados con el uso de Aditivos Alimentarios en la formulación de alimentos de diferente tipo.

En una primera etapa, se crearon cuatro grupos de trabajo, con cuatro alumnos cada uno seleccionados de forma aleatoria. Se debe evitar la formación de grupos preconcebidos, para evitar el agrupamiento de alumnos acostumbrados a trabajar entre ellos que inconsciente presentarían roles pre-asignados, para de esta forma fomentar la interacción entre los alumnos miembros. A cada uno de estos grupos iniciales se les asigna la tarea de resolver un problema de tipo práctico, proponer de forma consensuada y razonada la adición de aditivos si fuera necesario y el por qué en un determinado alimento. En una segunda etapa cada uno de los miembros del grupo inicial se dividió en 4 grupos de expertos para que se formaran en una materia específica (ver esquema en Figura 1). Los cuatro expertos, procedentes de cada uno de los diferentes grupos iniciales, se forman durante este tiempo en un grupo concreto de aditivos alimentarios frecuentemente empleados en alimentos, como antioxidantes, conservantes, edulcorantes, y colorantes. Cada grupo de expertos se sitúa en los extremos del aula para no interferir entre ellos y el profesor va coordinando las actividades dentro de cada grupo de expertos, para orientar al grupo y dirigir su aprendizaje en aquellos temas clave. Durante esta etapa, la formación consiste en la consulta y discusión de los apuntes de clase, clasificando los aditivos por naturaleza, riesgo, frecuencia de uso, características físico-químicas y coste.

\section{Grupos de expertos}

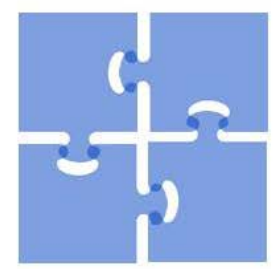

Antioxidantes

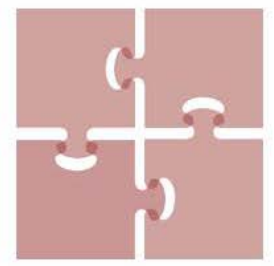

Conservantes

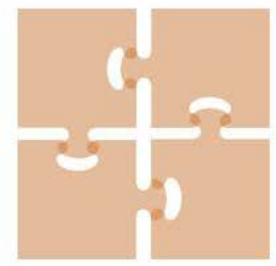

Edulcorantes

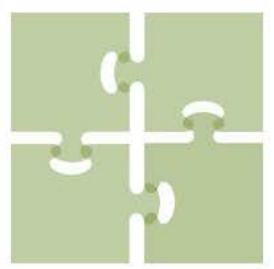

Colorantes

\section{Grupos de trabajo}
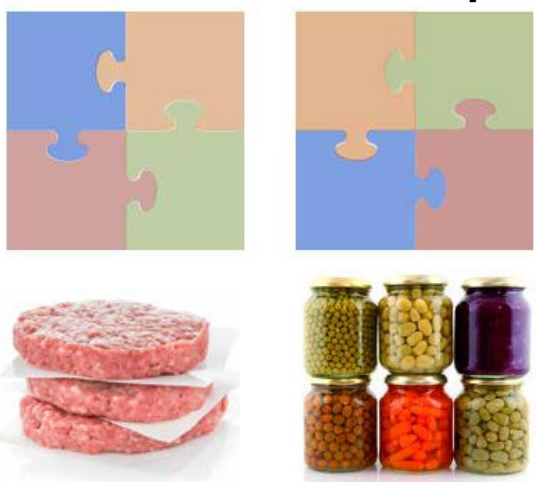

Vegetales
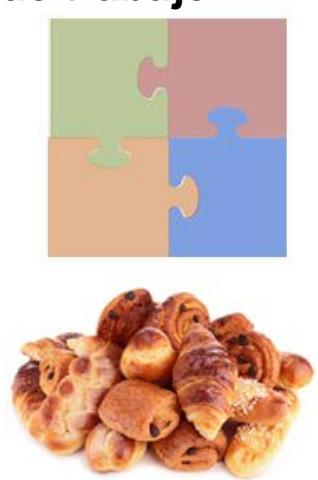

Bolleria
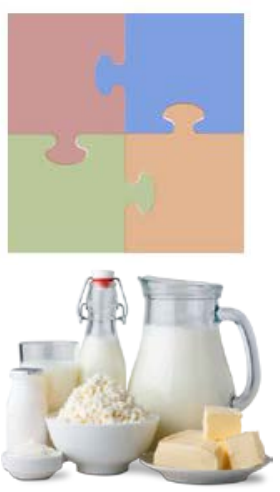

Lácteos

Fig. 1 Esquema de los grupos de expertos y de trabajo seleccionados en la actividad propuesta. 
Tras la primera sesión de tutoría, los expertos en aditivos buscan información adicional fuera del aula para complementar los conocimientos necesarios para poder ser empleados en la segunda tutoría. Esta búsqueda de información se realiza tanto por bibliografía reglada (libros, revistas, periódicos), como no reglada (consulta en Internet, foros, redes sociales, etc.). Además se hace especial hincapié en la evaluación de los ingredientes y aditivos que contienen los alimentos que los alumnos tengan en sus casas, y se propone la realización de trabajos de investigación con visitas a supermercados y tiendas locales para relacionar los diferentes aditivos estudiados en clase con ciertos grupos de alimentos. Durante las dos semanas que transcurren entre tutorías se hizo un seguimiento de la actividad para fomentar y promover esta búsqueda activa de etiquetas de alimentos. Este seguimiento se realizó durante el transcurso de clases de teoría.

La tercera fase se realizó el segundo día de tutoría. En ella, los expertos que se han formado en cada tipo de aditivos alimentarios se reagrupa en los cuatro grupos de trabajo iniciales para abordar la resolución del problema formulado el primer día de tutoría. La primera actividad a realizar consiste en la identificación y discusión de cada uno de los grupos de aditivos que contienen alimentos elaborados del siguiente tipo: productos cárnicos, vegetales envasados, panadería y bollería, y lácteos (ver Figura 1). Para la realización de esta actividad los alumnos deben de llevar a clase etiquetas de alimentos (o fotografías) recopiladas en los días previos a la realización de la segunda tutoría. En esta etapa, los alumnos proceden a realizar una evaluación del tipo de aditivos que llevan los alimentos, el por qué, posibles riesgos toxicológicos, consecuencias de su retirada de la formulación, propuesta de posibles sustitutos, y búsqueda de efectos sinérgicos. La Figura 2 muestra un ejemplo de las etiquetas originales que aportaron los estudiantes. Finalmente, cada grupo propone el tipo de aditivos a emplear en el alimento problema propuesto, su justificación, y si existe algún tipo de alternativa al empleo de los aditivos alimentarios propuestos.

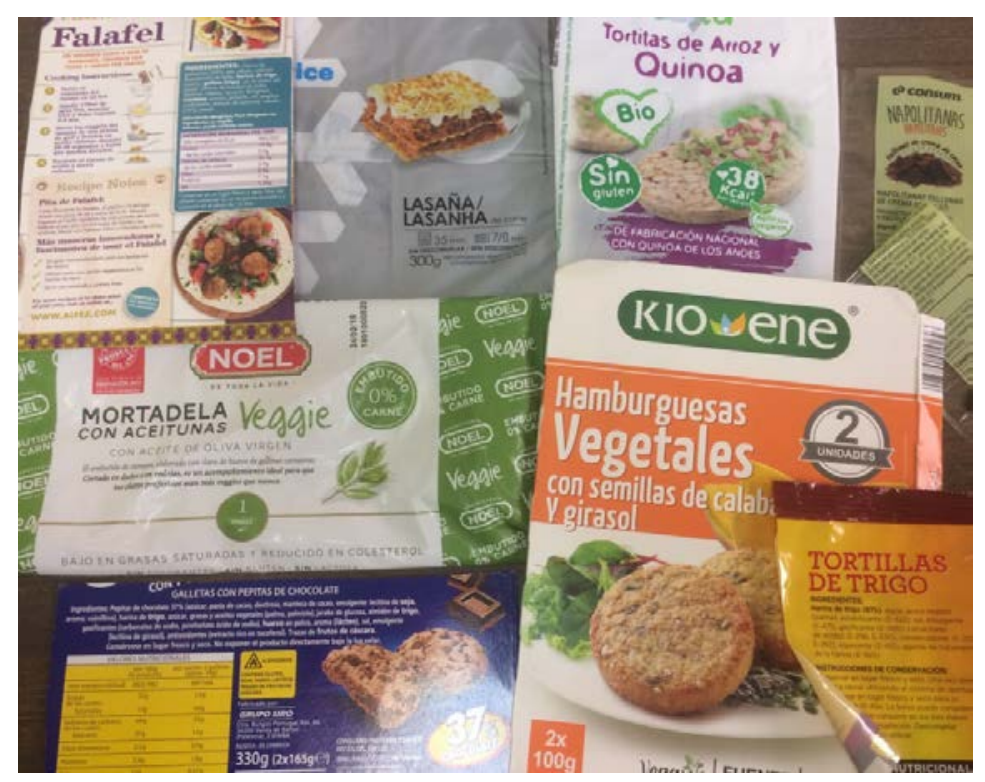

Fig. 2. Ejemplo etiquetas de alimentos recopiladas por los alumnos.

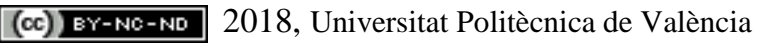

Congreso IN-RED (2018) 


\section{Resultados}

\subsection{Resultados de aprendizaje}

Con la tarea propuesta se pretende incitar al alumnado al empleo de herramientas cooperativas para facilitar la resolución de problemas. Concretamente se pretende desarrollar las siguientes competencias y habilidades sociales que figuran en la guía docente de la asignatura, como 1) capacidad de aplicar los conocimientos a la práctica; 2) razonamiento crítico que les permita emitir juicios argumentados y defenderlos con rigor y tolerancia; y 3) capacidad de trabajar en grupo, responsabilidad compartida, gestión de conflictos, y propuesta de soluciones objetivas. Adicionalmente, con el desarrollo de la actividad el alumno afianza los conceptos teóricos impartidos en clase, los complementa con información adicional y los pone en práctica. Debido a la temporalidad de la actividad, que se realiza a mitad de curso, el problema propuesto se centrará en aquellas familias de aditivos alimentarios más frecuentemente empleadas, que además se imparten en los primeros temas de la asignatura, como antioxidantes, antimicrobianos, edulcorantes y colorantes. En la primera sesión el estudiante repasa los conceptos teóricos y los amplía mediante la consulta de los apuntes de clase, consulta de libros especializados, o información adicional en Internet. En este sentido, resulta relevante el empleo de redes sociales y páginas de Internet para la búsqueda de contenidos adicionales, ya que esta información no reglada permite a los grupos de expertos discutir la veracidad o falsedad parcial de la información que está disponible en la red, lo que contribuye a fomentar su capacidad crítica.

En la segunda sesión de la actividad, lo grupos iniciales se reagrupan con cuatro expertos, ya formados, en cada uno de los aditivos alimentarios a evaluar. Cada grupo de trabajo comenzó a evaluar, discutir y justificar los aditivos presentes en las distintas etiquetas de alimentos suministradas por el profesor y también por los mismos alumnos. Durante esta etapa, los alumnos ven cuales son los aditivos que se emplean más frecuentemente y deben intentar relacionar ciertos aditivos específicos para grupo de alimentos concretos.

Finalmente, se les propone formular los aditivos que debe llevar un alimento concreto para que el grupo decida de una manera consensuada, razonada y justificada los productos químicos que se les debe añadir para ampliar su vida útil. En esta etapa del proceso cada miembro experto del grupo debe justificar y defender la presencia de su tipo de aditivo en la formulación final del alimento, lo que contribuye a la aparición de conflictos, que se deben resolver de forma consensuada para conseguir el mejor producto final. La resolución de conflictos y toma de decisiones comunes son competencias que resultarán de gran utilidad para el desarrollo de la carrera profesional de los alumnos.

\subsection{Evaluación de la actividad}

El aprendizaje por trabajo cooperativo se incorporó a la asignatura de Aditivos Alimentarios en el curso 2016-2017. En este primer año se hizo una evaluación del proceso de aprendizaje meramente cualitativa, y no se disponía de indicadores cuantitativos para asegurar que el alumno conseguía trabajar de forma cooperativa. Para la evaluación de 
dicha actividad se ha empleado una evaluación de tipo mixta con una parte formativa que valoró solo el proceso de aprendizaje (trabajo cooperativo) y otra sumativa para valorar la viabilidad y adecuación del resultado al problema propuesto. La contribución de cada una de estas partes será del $50 \%$. La evaluación de la parte formativa se realizó a través de la autoevaluación del alumno mediante el empleo de una rúbrica para conocer lo útil que ha sido esta herramienta para fomentar el trabajo cooperativo. La Tabla 1 muestra la rúbrica empleada con 7 criterios a evaluar en cuatro niveles. Los criterios fueron 1) Participación grupal, 2) Roles dentro del grupo, 3) Responsabilidad compartida, 4) Delegación de responsabilidad, 5) Calidad de interacción, 6) Calidad de las fuentes, y 7) ¿̇Se han cumplido los objetivos del equipo?. Mientras que el cumplimiento de cada criterio se evaluó como excepcional, admirable, aceptable o mejorable, puntuables con 10, 6, 4 y 0 puntos respectivamente. Se consideró que se ha trabajado de forma cooperativa cuando la nota final (la suma de todos los puntos obtenidos en cada criterio dividido por el número de criterios) era superior a 5 puntos. La evaluación de la parte formativa la realizó el profesor tras comprobar el resultado al problema propuesto y su correcta justificación.

El grado de cooperación conseguido en el trabajo en equipo propuesto se muestra en la Figura 3, con los resultados obtenidos a través de la autoevaluación por medio de la rúbrica anteriormente mencionada. En general la mayoría de criterios mostraron una respuesta favorable respecto al trabajo cooperativo, sobre todo el criterio 1 referente a la participación grupal con un 80.6 \% de puntuaciones excepcionales. Sin embargo, cabe destacar la menor puntuación del criterio 4 (delegación de responsabilidad) en el que un $51.6 \%$ valuaron como excepcional, mientras que un $41.9 \%$ como admirable. Esto se puede deber a que cada estudiante se hace responsable de la información que se le ha asignado buscar, pero en muchos casos no se identifica adecuadamente qué información es necesaria y relevante para el grupo y cual no. Por lo que se debe trabajar y hacer más hincapié en que la responsabilidad es compartida por todos los miembros del grupo y no existe ningún tipo de jerarquía con miembros con más responsabilidad o funciones que otros. Otro aspecto a tener en cuenta está relacionado con el criterio 6 (calidad de las fuentes), en el que a pesar que mayoritariamente se ha evaluado como excepcional (67.7\%) existe un porcentaje relativamente elevado de resultados aceptables (12.9\%), e incluso mejorable (3.2 \%). Este resultado es relevante, ya que a pesar de no ser mayoritario, existe un número determinado de alumnos que solo utilizó los apuntes de clases para realizar la tarea propuesta o incluso en algún caso no se utilizó ninguna fuente, mostrando una falta de interés en realizar esta actividad. Los aspectos que han tenido una menor puntuación se intentarán mejorar en el próximo curso, principalmente aquellos relacionados con la responsabilidad compartida del grupo y la falta de interés. De todos modos, se considera que los alumnos realizaron mayoritariamente la tarea propuesta mediante trabajo en grupo cooperativo de una manera muy satisfactoria. Además, el empleo de la rúbrica propuesta nos permite identificar aquellos puntos del proceso donde tenemos que hacer un esfuerzo extra tanto en el momento inicial en el que se plantea la tarea como en la supervisión del profesor durante su ejercicio. 
Tabla 1. Rúbrica propuesta para evaluar la capacidad de cooperación del grupo para solucionar un problema propuesto.

\begin{tabular}{|c|c|c|c|c|c|}
\hline & $\begin{array}{l}\text { Excepcional } \\
\text { (10 puntos) }\end{array}$ & $\begin{array}{l}\text { Admirable } \\
\text { (6 puntos) }\end{array}$ & $\begin{array}{l}\text { Aceptable } \\
\text { (4 puntos) }\end{array}$ & $\begin{array}{l}\text { Mejorable } \\
(0 \text { puntos })\end{array}$ & Total \\
\hline $\begin{array}{l}\text { Participación } \\
\text { grupal }\end{array}$ & $\begin{array}{l}\text { Todos los } \\
\text { estudiantes } \\
\text { participan } \\
\text { activamente en } \\
\text { la actividad. }\end{array}$ & $\begin{array}{l}\text { La mayoría de los } \\
\text { estudiantes } \\
\text { participan } \\
\text { activamente. }\end{array}$ & $\begin{array}{l}\text { Al menos la mitad } \\
\text { de los estudiantes } \\
\text { presentan ideas } \\
\text { propias. }\end{array}$ & $\begin{array}{l}\text { No existe } \\
\text { participación } \\
\text { activa. }\end{array}$ & \\
\hline $\begin{array}{l}\text { Roles dentro del } \\
\text { grupo }\end{array}$ & $\begin{array}{l}\text { Cada estudiante } \\
\text { tiene un rol } \\
\text { asignado bien } \\
\text { definido. }\end{array}$ & $\begin{array}{l}\text { Cada estudiante } \\
\text { tiene un rol } \\
\text { asignado, pero no } \\
\text { está claramente } \\
\text { definido o no es } \\
\text { consistente. }\end{array}$ & $\begin{array}{l}\text { Hay roles } \\
\text { asignados a los } \\
\text { estudiantes, pero } \\
\text { no se adhieren } \\
\text { consistentemente a } \\
\text { ellos. }\end{array}$ & $\begin{array}{l}\text { No hay ningún } \\
\text { esfuerzo de } \\
\text { asignar roles a } \\
\text { los miembros. }\end{array}$ & \\
\hline $\begin{array}{l}\text { Responsabilidad } \\
\text { compartida }\end{array}$ & $\begin{array}{l}\text { Todos comparten } \\
\text { por igual la } \\
\text { responsabilidad } \\
\text { sobre la tarea }\end{array}$ & $\begin{array}{l}\text { La mayor parte de } \\
\text { los miembros del } \\
\text { grupo comparten } \\
\text { la responsabilidad } \\
\text { en la tarea }\end{array}$ & $\begin{array}{l}\text { La responsabilidad } \\
\text { es compartida por } \\
\text { la mitad de los } \\
\text { integrantes del } \\
\text { grupo }\end{array}$ & $\begin{array}{l}\text { La } \\
\text { responsabilidad } \\
\text { recae en una sola } \\
\text { persona }\end{array}$ & \\
\hline $\begin{array}{l}\text { Delegación de } \\
\text { responsabilidad }\end{array}$ & $\begin{array}{l}\text { Cada estudiante } \\
\text { en el grupo puede } \\
\text { explicar qué } \\
\text { información es } \\
\text { necesaria para el } \\
\text { grupo y qué } \\
\text { información es } \\
\text { responsable de } \\
\text { localizar. }\end{array}$ & $\begin{array}{l}\text { Cada estudiante } \\
\text { en el grupo puede } \\
\text { explicar qué } \\
\text { información es } \\
\text { responsable de } \\
\text { localizar. }\end{array}$ & $\begin{array}{l}\text { Cada estudiante en } \\
\text { el grupo puede, } \\
\text { con la ayuda de } \\
\text { sus compañeros, } \\
\text { explicar qué } \\
\text { información es } \\
\text { responsable de } \\
\text { localizar. }\end{array}$ & $\begin{array}{l}\text { Uno o más } \\
\text { estudiantes en el } \\
\text { grupo no pueden } \\
\text { explicar qué } \\
\text { información } \\
\text { ellos son } \\
\text { responsables de } \\
\text { localizar. }\end{array}$ & \\
\hline $\begin{array}{l}\text { Calidad de } \\
\text { interacción }\end{array}$ & $\begin{array}{l}\text { Todos los } \\
\text { alumnos se } \\
\text { expresan } \\
\text { adecuadamente, } \\
\text { saben escuchar y } \\
\text { se respetan los } \\
\text { puntos de vista y } \\
\text { opiniones de los } \\
\text { demás. }\end{array}$ & $\begin{array}{l}\text { La mayor parte de } \\
\text { los alumnos se } \\
\text { expresa } \\
\text { adecuadamente, } \\
\text { sabe escuchar y } \\
\text { respeta los puntos } \\
\text { de vista y } \\
\text { opiniones de los } \\
\text { demás. }\end{array}$ & $\begin{array}{l}\text { Una minoría de los } \\
\text { alumnos se } \\
\text { expresa } \\
\text { adecuadamente, } \\
\text { sabe escuchar y } \\
\text { respeta los puntos } \\
\text { de vista y } \\
\text { opiniones de los } \\
\text { demás. }\end{array}$ & $\begin{array}{l}\text { Existe muy poca } \\
\text { interacción, } \\
\text { conversación } \\
\text { muy breve, } \\
\text { distracciones o } \\
\text { desinterés. }\end{array}$ & \\
\hline $\begin{array}{l}\text { Calidad de las } \\
\text { fuentes }\end{array}$ & $\begin{array}{l}\text { Se han utilizado } \\
\text { los apuntes de la } \\
\text { signatura y se han } \\
\text { ampliado en } \\
\text { búsquedas por } \\
\text { Internet y } \\
\text { legislación } \\
\text { oficial. }\end{array}$ & $\begin{array}{l}\text { Se han utilizado } \\
\text { los apuntes de la } \\
\text { signatura y se han } \\
\text { ampliado en } \\
\text { búsquedas por } \\
\text { Internet. }\end{array}$ & $\begin{array}{l}\text { Se han utilizado } \\
\text { los apuntes de la } \\
\text { asignatura } \\
\text { exclusivamente. }\end{array}$ & $\begin{array}{l}\text { No se ha } \\
\text { utilizado ningún } \\
\text { tipo de fuente. }\end{array}$ & \\
\hline $\begin{array}{l}\text { ¿Se han } \\
\text { cumplido los } \\
\text { objetivos del } \\
\text { equipo? }\end{array}$ & $\begin{array}{l}\text { Si, con gran } \\
\text { probabilidad de } \\
\text { implantación. }\end{array}$ & $\begin{array}{l}\text { Sí, con poca } \\
\text { probabilidad de } \\
\text { implantación. }\end{array}$ & Parcialmente. & No. & \\
\hline & & & & \multicolumn{2}{|l|}{$\begin{array}{l}\text { Nota final = } \\
\text { (puntos / } 7 \text { ) }\end{array}$} \\
\hline
\end{tabular}

Para finalizar, el rendimiento académico conseguido fue de $3.3 \%$ matrícula de honor, $6.7 \%$ sobresaliente, $56.7 \%$ notable, $30.0 \%$ aprobado, $3.3 \%$ suspenso, y $9.1 \%$ no presentado. El número de aprobados en la asignatura es inferior al curso 2016-2017, pero se debe tener en cuenta que aún falta realizar la segunda convocatoria de dicha asignatura, por lo que es probable que a final de curso el porcentaje de aprobados sea superior. 


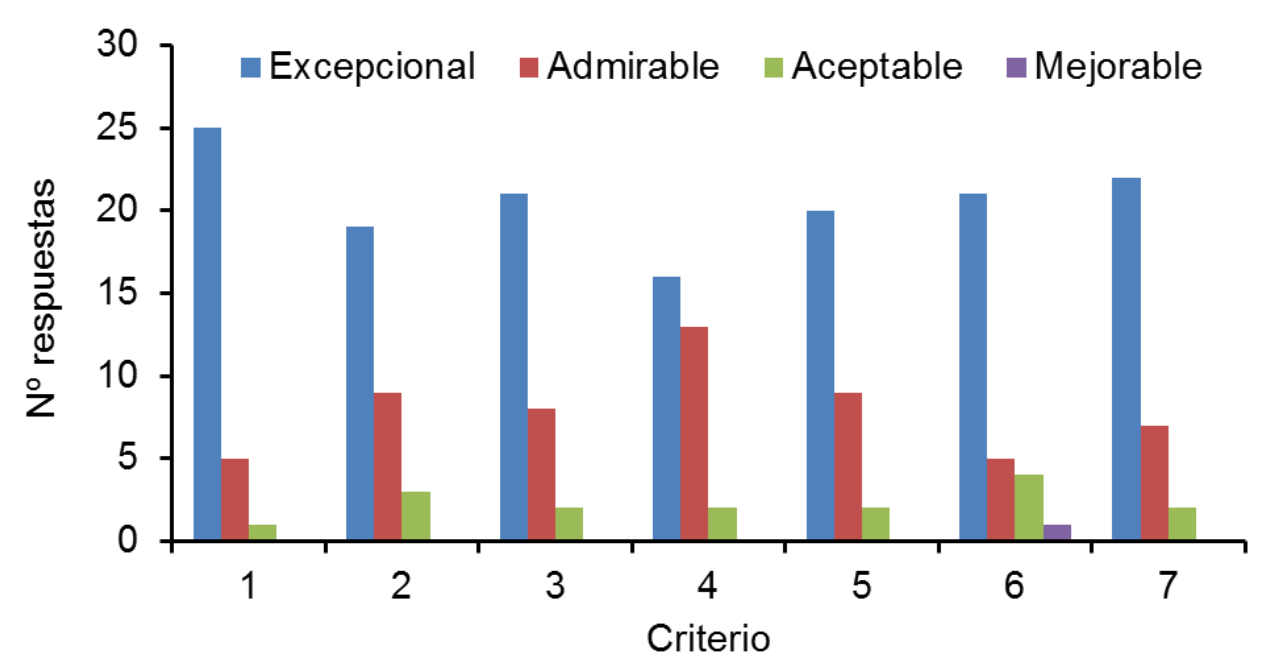

Fig. 3. Resultados obtenidos para cada criterio de la rúbrica.

Adicionalmente, al finalizar la tarea propuesta los alumnos rellenaron un cuestionario para conocer su grado de satisfacción y opinión acerca del trabajo cooperativo en grupo. A los alumnos se le hicieron cuatro preguntas cortas: 1) ¿Has realizado con anterioridad alguna actividad para fomentar el trabajo cooperativo?, 2) ¿Crees que se ha incrementado tu capacidad para trabajar de forma cooperativa?, 3) ¿Qué opinas del trabajo cooperativo?, y 4) Indica tu grado de satisfacción con la actividad propuesta. La Figura 4 muestra las respuestas a las cuatro preguntas efectuadas. Como se observa en la figura el 45 \% de los alumnos habían realizado con anterioridad actividades que implicaban trabajo cooperativo y un 19\% nunca. Sin embargo, lo más relevante es el 36\% de alumnos que dudan o no están seguros de haber realizado dichas tareas. Algo similar ocurre con la percepción para trabajar de forma cooperativa, en el que $68 \%$ duda sobre si se ha incrementado o no su capacidad de trabajo, mientras que un $32 \%$ afirma rotundamente que sí lo ha hecho. Eso refleja claramente la confusión entre trabajo en grupo y trabajo en grupo cooperativo cuya barrera no está claramente delimitada para el alumno y su percepción es ambigua. Por lo que para el próximo año, se hará más hincapié en la explicación del trabajo cooperativo y las diferencias fundamentales con trabajo individual y competitivo. Respecto a la utilidad del trabajo cooperativo un $77 \%$ lo considera muy útil y un $23 \%$ cree que puede tener alguna utilidad, siendo el grado de satisfacción para un $42 \%$ es adecuado y para un 52 $\%$ medio-alto.

Debido a la respuesta favorable y el interés mostrado por los alumnos se continuará realizando la presente actividad el curso que viene, incorporando ligeros cambios para asegurar la correcta aplicación del trabajo en grupo y mejorar la motivación entre aquellos estudiantes de baja participación. Además, el desarrollo de este tipo de actividades nos permite trabajar competencias transversales que son difíciles de incorporar y no se aplican habitualmente en el aula.

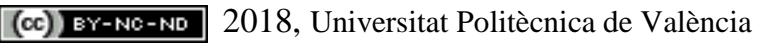

Congreso IN-RED (2018) 

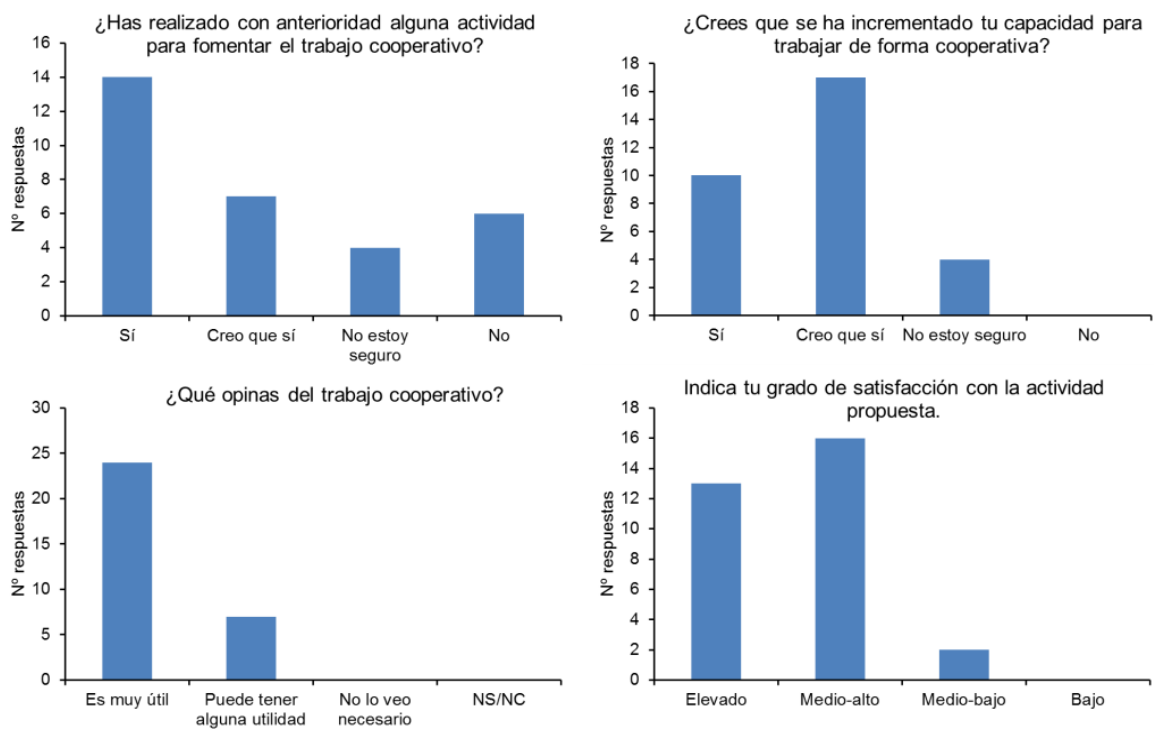

Fig. 4. Cuestionario de opinión de la actividad propuesta.

\section{Conclusiones}

El empleo de una dinámica de trabajo cooperativo en grupo, basada en el puzle de Aronson, se ha incorporado por segundo año consecutivo a las tutorías de la asignatura de Aditivos Alimentarios del Grado de Ciencia y Tecnología de los Alimentos de la Universitat de València. A través del empleo de esta metodología se trabajan en el aula competencias relacionadas con el trabajo cooperativo, se desarrollan habilidades sociales y de comunicación, y se fomenta la resolución de conflictos del grupo. En este segundo año, se ha empleado una rúbrica para evaluar el proceso de trabajo cooperativo que ha resultado de mucha utilidad para asegurar la consecución del objetivo propuesto. Adicionalmente, los resultados obtenidos a través de dicha rúbrica nos han permitido en algunos casos detectar fallos de ejecución, que se intentarán mejorar en la aplicación de esta dinámica el curso que viene. En este sentido, se debe fomentar que el alumno perciba que la responsabilidad en la resolución del problema propuesto debe recaer en todos sus miembros. Adicionalmente, se realizó una encuesta para evaluar el grado de utilidad y satisfacción para el alumno en la consecución de dicha tarea. El grado de satisfacción fue muy elevado entre el alumnado, así como la visión positiva de las técnicas de trabajo cooperativo. Sin embargo, se ha identificado que el alumno no percibió con claridad las diferencias entre trabajo en grupo y trabajo cooperativo en grupo, ya que la encuesta ha reflejado dudas a la hora de identificar si la actividad propuesta ha mejorado su capacidad de trabajar cooperativamente. Finalmente, desde el punto de vista del profesor, el balance final ha sido muy positivo ya que se ha fomentado el desarrollo de habilidades sociales y el trabajo en grupos cooperativos para la resolución de problemas, todo ello en un marco estrechamente ligado a los conocimientos teóricos-técnicos que se imparten en la asignatura de aditivos alimentarios. 


\section{Referencias}

JOHNSON, D. y JOHNSON, R. (1991). Learning together and alone. Cooperative, competitive and individualistic learning. Needham Heights, Allyn and Bacon.

JOHNSON, D.W., JOHNSON, R.T., y HOLUBEC, E.J. (1994). Cooperative Learning in the Classroom. Association For Supervision and Curriculum Development, Virginia, USA.

KAGAN, S. (1994). Cooperative Learning. San Clemente, CA, USA.

MARTÍNEZ, J. y GÓMEZ, F. (2010). La técnica puzzle de Aronson: descripción y desarrollo, en Arnaiz, P., Hurtado, M.D., y Soto, F.J. 25 Años de Integración Escolar en España: Tecnología e Inclusión en el ámbito educativo, laboral y comunitario. Murcia: Consejería de Educación, Formación y Empleo.

TRAVER, J. A. y GARCÍA, R. (2004). "La enseñanza-aprendizaje de la actitud de solidaridad en el aula: una propuesta de trabajo centrada en la aplicación de la técnica puzzle de Aronson” en Revista Española de Pedagogía, 229, 419.

ESTEVE-TURRILLAS, F.A. y ARMENTA, S. (2017). "Empleo de la estrategia puzle de Aronson para fomentar la cooperación en grupos” en Congreso In-Red 2017, Valencia. Disponible

http://dx.doi.org/10.4995/INRED2017.2017.6867> 